Eur J Clin Chem Clin Biochem

1995; 33:839-845

(c) 1995 Walter de Gruyter \& Co. Berlin - New York

\title{
Peripheral Blood Monocyte Counting: Towards a New Reference Method
}

\author{
By Wolfgang Hübl ${ }^{1}$, Sylvia Andert ${ }^{1}$, Angelika Erath ${ }^{1}$, Alexander Lapin ${ }^{2}$ and Peter M. Bayer ${ }^{1}$ \\ ${ }^{1}$ Institut fiir Klinische Chemie, Wilhelminenspital, Wien, Austria \\ 2 Zentrallabor, Allgemeine Poliklinik der Stadt Wien, Wien, Austria
}

(Received May 9/September 1, 1995)

Dedicated to Prof. Dr. Erich Kaiser on the occasion of his 70th birthday

Summary: Flow cytometric enumeration of monocytes stained with fluorescence-labelled monoclonal antibodies has been proposed as a possible reference method for monocyte counting. We compared precision and accuracy of monocyte counting of the Coulter STKS, the Cobas Argos 5 Diff, the 800-cell manual differential, and the Coulter Epics Profile II flow cytometer using double-staining with fluorescence-labelled monoclonal antibodies (CD45FITC and CD14-PE).

Precision: STKS, Argos and Profile II achieved a precision analogous to a 3423-, 1298-, and 11089-cell differential, respectively, confirming the superiority of automated methods.

Accuracy (136 normal and abnormal samples): Correlation of automated methods with the manual differential was good (STKS: $r=0.934$, Argos 5 Diff: $r=0.808$, Profile II: $r=0.924$; Spearman's rank correlation coefficient). The mean relative STKS monocyte result was $0.52 \pm 1.63 \%$ (mean $\pm \mathrm{SD}$ ) higher than the manual differential, whereas the Argos 5 Diff results were $1.22 \pm 2.51 \%$ lower $(\mathrm{p}<0.001)$. Profile II results showed a small bias against the manual differential $(-0.18 \pm 1.44 \%, \mathrm{p}<0.05)$.

Analysing 135 healthy adult subjects on the Profile II, males were found to have a higher mean monocyte count (relative count: $6.95 \pm 1.43 \%$ vs. $5.86 \pm 0.98 \%$; absolute count: $0.48 \pm 0.15 \times 10^{9} / 1$ vs. $0.39 \pm 0.11 \times 10^{9} / 1$, $\mathrm{p}<0.001$ ) and a higher and wider normal range than females (relative count: 4.97 to $9.78 \%$ vs. 4.26 to $7.81 \%$, absolute count: 0.30 to $0.84 \times 10^{9} / 1$ vs. 0.25 to $\left.0.65 \times 10^{9} / 1\right)$.

Flow cytometry based on fluorescence-labelled monoclonal antibodies for monocyte enumeration seems an efficient tool to evaluate the monocyte counting performance of haematology analysers and an ideal successor to the manual differential as reference method for monocyte counting.

\section{Introduction}

Evaluations of the differential leukocyte count of haematology analysers have often yielded satisfactory results for neutrophils, lymphocytes, and eosinophils, whereas the performance of monocyte counting has been disappointing $(1-8)$, even when studying only normal samples $(9,10)$. The correlation with the reference method was frequently poor and both accuracy and precision worse than for other leukocyte classes. Although monocytes represent a relatively small leukocyte class, this cannot be the main reason for this, as the less frequent eosinophils usually showed good results $(1-8,11)$. The morphological variety of monocytes definitely poses problems for automated differentiating techniques, another serious problem being lack of an appropriate reference method. The value of the manual 400 -cell differential, which is still used as reference in monocyte counting (12), is diminished by subjectivity of the examiner (13) and a low precision for smaller cell populations 
(14). To correctly assess monocyte counting performance, investigators evaluating the differential leukocyte count of haematology analysers have begun to employ flow cytometric methods using monocyte-specific monoclonal antibodies and have proposed this technique as an alternative reference method $(9,15,16)$.

In the present study, we investigated the monocyte counting performance of the Coulter STKS (Coulter Corp., Hialeah, FL) and the Cobas Argos 5 Diff (Hoffmann La Roche, Montpellier, France) haematology analysers by comparing them with an 800-cell manual differential following a modified NCCLS protocol. We also evaluated a flow cytometric method based on dual staining of leukocytes with monocyte-specific fluorescencelabelled monoclonal antibodies to test its suitability as a new reference method for monocyte counting. A normal range for this method was established. As superior precision is an important criterion for any reference method, precision was extensively investigated by establishing precision profiles for all methods under study.

\section{Materials and Methods}

\section{Instruments}

All instruments were used with the reagents recommended and supplied by their manufacturers. The Coulter STKS with VCS technology classifies cells by measuring low-frequency impedance, highfrequency conductivity and laser light scatter. The STKS typically differentiates 8192 events in all but severely leukopenic samples. The software version used during the study was 1G1. The Cobas Argos 5 Diff measured impedance and optical transmission of leukocytes after staining of eosinophils. Basophils are detected in a specific channel after lysis of all other cells. In contrast to the STKS, the Argos differentiates a fixed volume of diluted whole blood. Software version 3.37 was used throughout the study.

The Coulter Epics Profile II flow cytometer measured forward scatter, side scatter, and two-colour fluorescence. It was programmed to analyse 20000 events. Dual staining with fluorescence-labelled monoclonal antibodies was performed as follows:

Hundred $\mu$ l of EDTA anti-coagulated whole blood were incubated for 15 minutes at room temperature with a combination of $20 \mu \mathrm{l}$ CD45-FITC (IT, Immunotech, Paris, France, anti-leukocyte antibody, clone J33) and $20 \mu \mathrm{l} \mathrm{CD14-PE} \mathrm{(Immunotech,} \mathrm{anti-monocyte}$ antibody, clone RM052). Subsequently, erythrocytes were lysed by adding $2 \mathrm{ml}$ of an ammonium chloride-based lysing solution (AnDer-Grub Bio Research, Kaumberg, Austria). After two washing steps, samples were analysed. Monocytes were identified according to their CD14 antigen expression in the CD45-CD14 histogram; the CD14 weak monocytes were included.

\section{Sample collection}

Venous blood was drawn into standard 3-ml $\mathrm{K}_{3}$ EDTA tubes. The samples were maintained at room temperature and tested between 1 and 3 hours after collection in the automatic sampling mode on the STKS and the Cobas Argos. The time interval between measurements on the two instruments was always less than one hour Samples were prepared for flow cytometry within 4 hours of collection. Three blood spins were prepared for each specimen (Microx spinner, Omron, Tokyo, Japan). They were fixed and stained using a modified Wright method (Hema-Tek, Ames Auto- matic Stain, Miles Limited, Slough, UK). The sequence of application of the various systems was changed every day.

\section{Manual Differential}

The 800-cell manual differential was obtained as follows. First, a 400 -cell differential was carried out as described in the NCCLS Approved Standard H20-A (12): two clínical pathologists each performed a 200-cell differential on separate blood spins and the results were averaged. If the difference between the two manual counts exceeded the $95 \%$ confidence limits given by Rümke (14), a third spin was counted by an arbitrator and the results processed as recommended in the NCCLS guidelines (12). The 800-cell manual differential was then obtained by averaging the result of this 400-cell differential with that of another one performed by a technician to increase the precision of results.

\section{Accuracy}

Blood samples from 143 subjects of the daily routine were analysed. Thirty-one samples were normal as defined by the absence of any abnormal distributional or morphological haematological finding in the manual differential. Pathological samples were defined, according to the NCCLS recommendations, as those showing abnormal distributional or morphological features in the manual differential (12). They came from 112 patients with various pathologies (viral and bacterial infection, liver cirrhosis, carcinoma, chronic renal failure, multiorgan failure, hyperthyreosis, diabetes, autoimmune diseases). No samples with monocyte counts $>25 \%$ were used as they might have unduly influenced correlation results to the extent of obscuring bad performance in the normal range.

Passing \& Bablok linear regression $(17,18)$ was applied to calculate slope and intercept, and Spearman's rank correlation coefficient ( $r$ ) was calculated. As a measure of deviation from the regression line, the $68 \%$ median distance (ma68) is given, which is the non-parametric analogue of the standard error of the estimate $s_{y x}$ of least squares linear regression. Friedmann's test was used to detect significant differences between methods or instruments, followed by Wilcoxon's signed rank test to identify the relevant groups. Passing \& Bablok regression and descriptive statistics were calculated with the Evalpak2 software (Boehringer Mannheim, Mannheim, Germany), non-parametric method comparison tests were performed with Statgraphics 4.0 (STSC, Rockville, MD). The criterion of statistical significance used for all tests was $\mathrm{p}<0.05$. Data are given as means $\pm \mathrm{SD}$.

\section{Reference range}

To define the reference range for the monoclonal antibody-based flow cytometric method, we analysed blood samples of 135 white adult subjects of Central European origin found to be healthy in a preventive medical checkup (69 males, 66 females, age 42.9 \pm 14.0 years, white blood count $\left.6.87 \pm 1.81 \times 10^{9} / 1\right)$. Blood was drawn in the morning after an overnight fast. Differences between males and females were tested for statistical significance with the Mann-Whitney U-test. Age and white blood count of males and females were not statistically different. Chi-square test was used to examine whether the data followed a normal distribution. The nonparametric normal range was described by giving the 2.5 and 97.5 percentiles.

\section{Precision}

Forty samples of the daily routine with different relative monocyte counts were analysed 15 times. The STKS was used in the manual mode; the Cobas Argos was operated in the autosampling mode as no differential for manually analysed samples was reported. The white blood cell counts of the samples varied between $4 \times 10^{9} / 1$ 


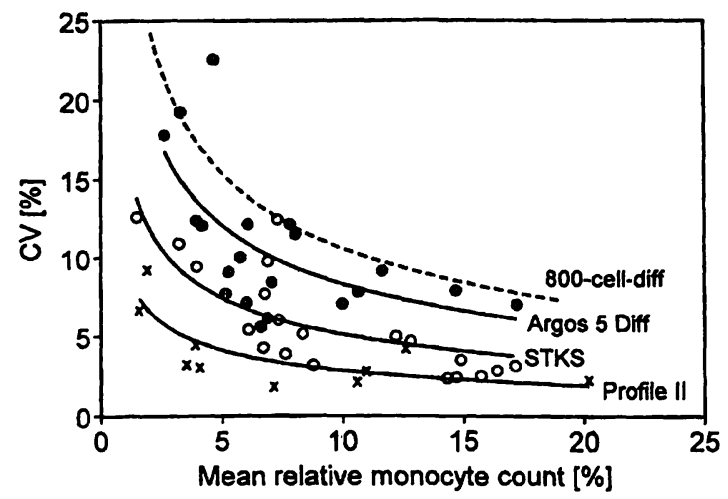

Fig. 1 Monocyte precision profiles: each data point represents the mean relative monocyte count ( $\mathrm{x}$-axis) and the coefficient of variation (y-axis) of one sample analysed 15 times. Curves were fitted to the data points of the respective instrument or were calculated for the 800-cell differential (dashed line) following the function for the coefficient of variation of proportions (STKS: open circles, Cobas Argos 5 Diff: solid circles, Profile II: $x$ ).

and $15 \times 10^{9} /$. The experiments were distributed over the whole evaluation period. To assess the precision of flow cytometry, 10 samples were prepared 15 times each. Evaluation of precision was performed as described previously (15). In brief, a precision profile was established by plotting the resultant coefficients of variation against the mean relative monocyte counts (fig. 1). The data points for each analyser where then fitted to a function describing the coefficient of variation of proportions (CVp):

$$
C V p(\%)=100 \times \frac{\text { SEp }}{p}
$$

SEp is the standard errror of proportions defining the confidence intervals for a single proportion (12):

$$
\operatorname{SEp}=\sqrt{\frac{p \times q}{n}}
$$

where: $\mathrm{n}=$ number of observed cells,

$\mathrm{p}=$ mean value (i.e., relative count in $\%$ ),

$\mathrm{q}=100-\mathrm{p}$.

As a result of the curve fitting the figure " $n$ " is obtained, which is equivalent to the number of theoretically differentiated leukocytes (i.e., the variable " $n$ " in the equation).

The optimal theoretical imprecision of the manual 800-cell differential was calculated using the function for the CVp. The curves fitted to the precision profiles of the instruments were compared based on the F test method (19). Calculations were performed using commercially available curve-fitting computer software based on the Marquardt algorithm (Fig-P for Windows, Biosoft, Cambridge, U.K.).

Day-to-day variability of flow cytometry was assessed conventionally by analysing whole blood control material (Ortho Abso-
luteControl, Ortho Diagnostic Systems Inc., Raritan, NJ, USA) on 20 consecutive days.

\section{Results \\ Precision}

The precision profiles show that, predictably, imprecision increased with decreasing monocyte percentages in all methods investigated (fig. 1), but they also demonstrate that the precision of both haemotology analysers can show large variations for different referencerange samples. This emphasises that precision studies based on replicate analysis of only one or few samples may totally misjudge analyser performance. The deviations of the Argos data points from the fitted curve were greater than those of the STKS or Profile II. The theoretical, minimal imprecision of the manual 800-cell differential was higher than that of the automated methods. The precision of the STKS was theoretically analogous to a 3423-cell differential and significantly exceeded that of the Cobas Argos 5 Diff, which corresponded to 1298-cell differential $(p<0.001)$. With a theoretical number of 11089 differentiated cells, the Profile II monocyte count significantly outperformed all other methods $(p<0.001)$. Investigation of day-to-day variability showed a coefficient of variation of $3.31 \%$ at a mean concentration of $12.39 \%\left(0.86 \times 10^{9} / 1\right)$.

\section{Accuracy}

The results of Passing \& Bablok regression are compiled in table 1 . Means and biases of monocyte results are

Tab. 1 Regression against manual differential: results of Passing \& Bablok regression analysis with the results of the manual differential used as $x$-variable $(n=136$, normal and abnormal samples; monocytes expressed as percent of leukocytes).

\begin{tabular}{llll}
\hline & STKS & Argos 5 Diff & Profile II \\
\hline Slope & 1.05 & $0.78^{*}$ & 0.94 \\
Intercept & 0.03 & 0.16 & 0.17 \\
ma68 & 0.86 & 1.16 & 0.85 \\
r (rank) & 0.934 & 0.808 & 0.924 \\
\hline
\end{tabular}

* significantly different from $1(p<0.05)$

a ma68 $=68 \%$ median distance

ferential (monocytes expressed as percent of leukocytes). Results given as means $\pm \mathrm{SD}$.
Tab. 2 Bias table: Mean relative monocyte count results and biases of haematology analysers. Bias expressed as relative monocyte count of test method minus the result of the manual dif-

\begin{tabular}{lllrr}
\hline & 800-cell diff. & STKS & Argos 5 Diff & Profile II \\
\hline Mean relative count & $8.15 \pm 3.79$ & $8.67 \pm 4.30$ & $6.94 \pm 3.86$ & $7.98 \pm 3.88$ \\
Bias & $0: 00 \pm 0.00$ & $0.52 \pm 1.63^{\mathrm{b}}$ & $-1.22 \pm 2.51^{\mathrm{b}}$ & $-0.18 \pm 1.44^{\mathrm{a}}$ \\
\hline
\end{tabular}

$\mathrm{p}<0.05$

b $p<0.001$ (bias significantly different from zero) 

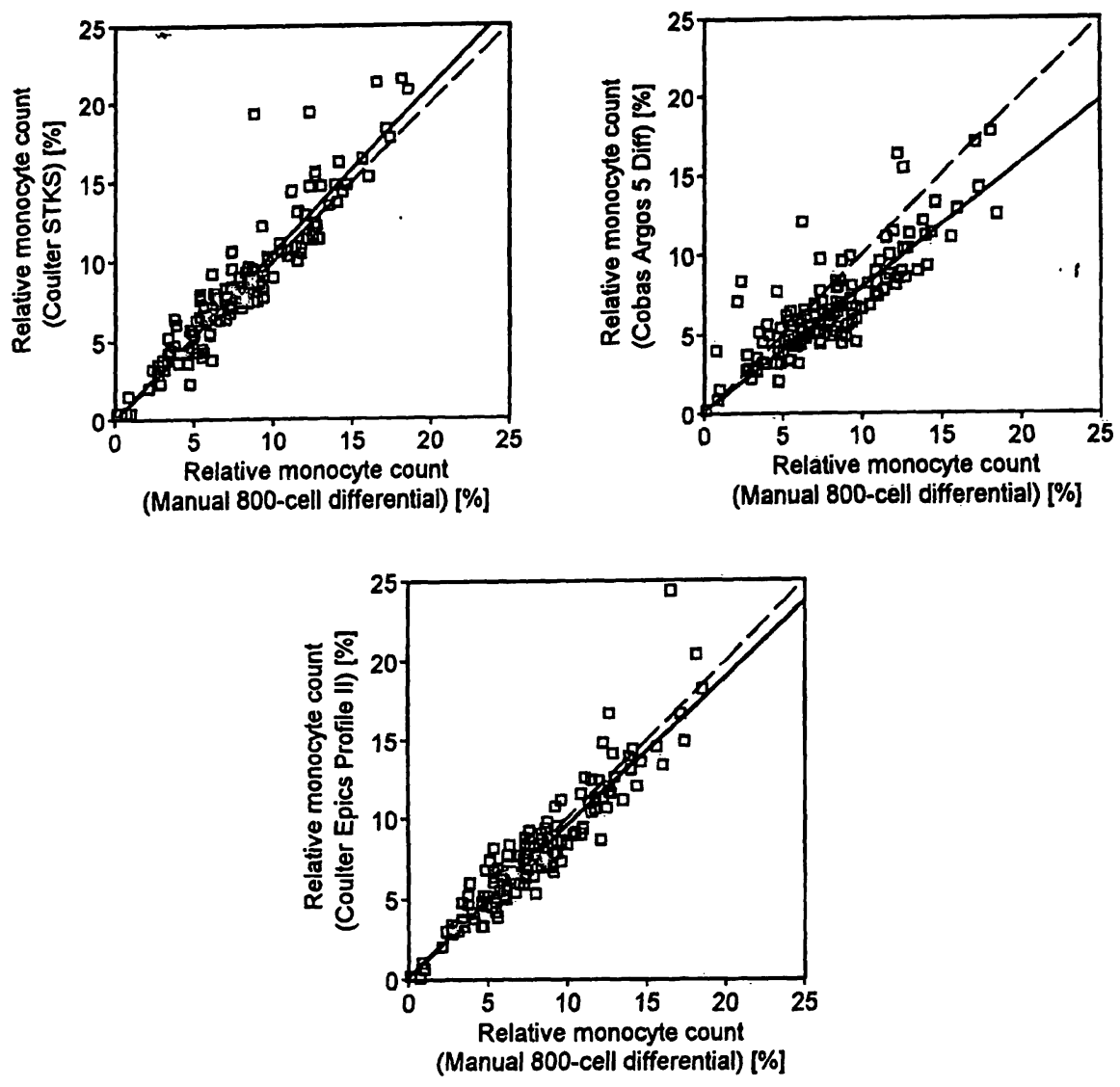

Fig. 2 Passing \& Bablok regression plots: the relative monocyte results of automated methods ( $y$-axes) are plotted against those of the manual 800 -cell differential $(n=136$; monocytes expressed as percent of leukocytes; solid line: Passing \& Bablok regression line, dashed line: identity line).

were excluded, as was one sample of a patient treated with granulocyte colony stimulating factor, which showed no distinct monocyte population on the Profile II.

\section{Reference range}

The monocyte results of all healthy subjects averaged $6.42 \pm 1.34 \%$ (absolute count: $0.44 \pm 0.14 \times 10^{9} / 1$ ) and showed a reference range of 4.45 to $9.30 \%$ ( 0.26 to 0.82 $\left.\times 10^{9} / 1\right)$. However, the distribution did not meet the criteria of a normal distribution. Age did not influence the monocyte count, but we found that male subjects had a significantly higher count and a wider range than fe-

Tab. 3 Regression against Profile II: Results of Passing \& Bablok regression analysis with the Profile II flow cytometry results used as $x$-variable $(n=136$, normal and abnormal samples; monocytes expressed as percent of leukocytes).

\begin{tabular}{lcc}
\hline & STKS & Argos 5 Diff \\
\hline Slope & $1.12^{*}$ & $0.87^{*}$ \\
Intercept & -0.26 & -0.33 \\
ma68 & 0.62 & 0.84 \\
r (rank) & 0.941 & 0.817 \\
\hline
\end{tabular}

* significantly different from $1(p<0.05)$ 
males (relative count: $6.95 \pm 1.43 \%$ vs. $5.86 \pm 0.98 \%$; absolute count: $0.48 \pm 0.15 \times 10^{9} / 1$ vs. $0.39 \pm 0.11$ $\times 10^{9} / 1, p<0.001$, fig. 3 ). Only the female relative counts were normally distributed. We therefore used non-parametric methods to describe the reference range for both groups, which would range from 4.97 to $9.78 \%$ $\left(0.30\right.$ to $\left.0.84 \times 10^{9} / 1\right)$ for males and from 4.26 to $7.81 \%$ $\left(0.25\right.$ to $\left.0.65 \times 10^{9} / 1\right)$ for females.

\section{Discussion}

\section{Precision}

We decided not to use the NCCLS-recommended procedure of duplicate analysis of the entire study population (12) to assess precision, as these results are strongly influenced by the size of the respective leukocyte class (1) and may fail to reveal precision performance in the infrequent high- or low-range samples. The precision profiles used in the present study describe precision performance over the whole relative count range, facilitate statistical comparison between different methods, and relate the performance to that of the manual differential. However, it must be noted that our curve-fitting function only allows for an influence of the relative but not of the absolute cell count, which is not ideal for the Cobas Argos, which, in contrast to the other methods, differentiates a fixed volume of blood rather than a fixed
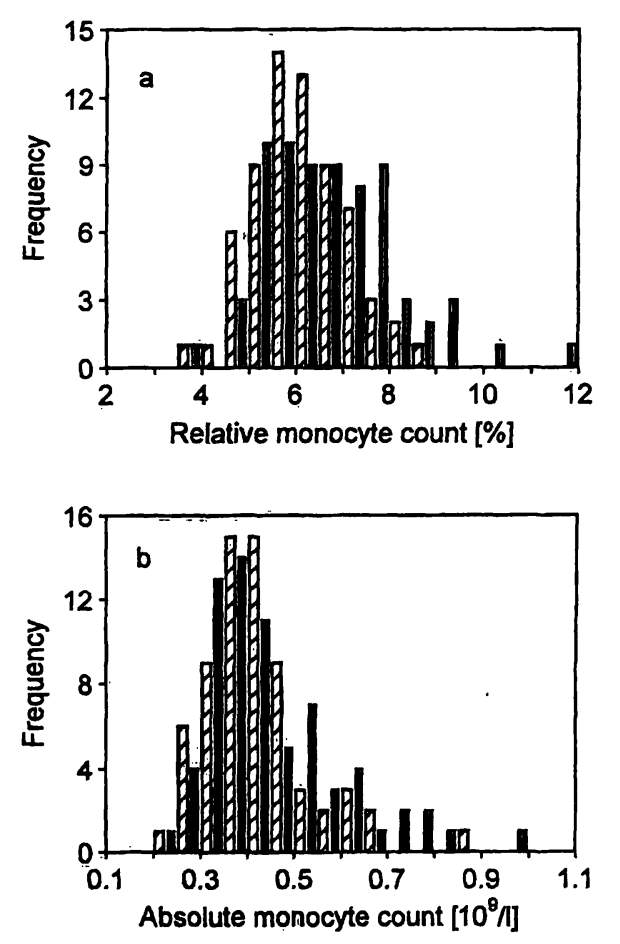

Fig. 3 The histogrammes show the distribution of the relative (a, classes of $0.5 \%$ ) and the absolute $\left(b\right.$, classes of $\left.0.05 \times 10^{9} / 1\right)$ monocyte counts in 69 males (solid bars) and 66 females (hatched bars). The mean female relative and absolute counts were significantly lower than those of the male subjects. number of leukocytes. This may be one reason for the larger deviations of the Argos data points from the fitted curve (fig. 1), although we moderated this effect by using only samples with a white blood cell count between $4 \times 10^{9} / 1$ and $15 \times 10^{9} / 1$.

The precision of both instruments was high and superior to that of the manual differential. The good precision of the STKS monocyte count is consistent with previous reports $(7,8)$. The precision of flow cytometry using fluorescence-labelled monoclonal antibodies on the Profile II reached more than half of the theoretical optimum of a 20000-cell differentiation despite a manual sample preparation procedure that included two washing steps.

Day-to-day variability was assessed with whole blood control material containing unfixed cells in a stabilising solution. As the material is primarily intended for the quality control of lymphocyte immunophenotyping, the leukocyte or monocyte concentration is not specified. Light scatter and fluorescence characteristics of leukocytes were somewhat different from normal samples, and separation of cell populations was generally not as clear. Nevertheless the results were satisfactory as dayto-day variability was only slightly higher than withinrun imprecision at the corresponding monocyte concentration.

Batch stability of the antibody was not tested, but a previous study showed an excellent agreement of flow cytometric monocyte results obtained with different CD14 and CD45 antibody clones, which suggested that the method was rather insensitive as to the antibody used (20).

\section{Accuracy}

Although problems have been reported for Passing \& Bablok regression analysis regarding proportional errors and hypothesis testing (21), the method was chosen for its superior resistance to outliers $(17,18,21)$. Therefore it is difficult to compare our results with those of least squares linear regression reported by other investigators. Based on the correlation coefficient (tab. 1), our correlation results of the STKS were better than in most other studies $(3,8,9,22,23)$. Most investigators comparing the Coulter VCS technology with microscopy observed a smaller STKS monocyte count $(1,3,10,23)$, while other authors reported a substantial influence of the software version used (22). Such an influence was also reported for other instruments (6). The Cobas Argos monocyte results correlated less closely with the 800-cell differential than those of the STKS. Moreover, the Argos showed a considerable mean negative bias and numerous outliers with a positive bias against the microscopic result. The mean negative bias against the manual dif- 
ferential is in contrast to a report by Bentley et al., who evaluated the Cobas Helios (Roche Diagnostic Systems, Inc., Branchburg, NJ, USA), which is very similar to the Argos, and found a positive mean bias (24), probably due to a different setting of discriminators in the Argos scattergramme. On the other hand, the high number of positive outliers observed in the present study is consistent with their report. In our study, most of these outliers were caused by insufficient separation of the monocyte population from the adjacent neutrophil population. Even a small overlap of this usually much larger population into the monocyte region can considerably increase the monocyte result.

Another study reported disastrous correlation results for the Argos monocyte count with a correlation coefficient of 0.386 and a slope of 4.2 when compared to a 400 cell manual differential (25), which is in contrast to the much better results observed in our study. We cannot give a definitive explanation for this discrepancy, but we believe that the choice of the blood film preparation technique is of pivotal importance for reliable microscopic monocyte results. The use of the spinning technique ensures even distribution of cells while microscopic results obtained with the wedge technique are often unreliable $(13,26,27)$.

In agreement with our previous results $(15,20)$ and with those of other investigators (8), we found a high correlation of the monoclonal antibody-based flow cytometric monocyte results with those of the manual differential. However, in contrast to our previous studies, we now observed a small negative bias of the Profile II monocyte counts against the manual differential. Although those studies included different monoclonal antibodies for monocyte labelling, we do not believe that this was the reason for the discrepancies. More likely, this was because the microscopic examination was performed by other individuals than in our previous studies. The correlation of the STKS monocyte count with the Profile II results (tab. 3) and the STKS bias against the Profile II were nearly identical with our previous results (15), emphasizing the objectivity and reproducibility of monoclonal antibody-based flow cytometric monocyte results.

\section{References}

1. Bentley SA, Johnson A, Bishop CA. A parallel evaluation of four automated hematology analysers. Am J Clin Pathol 1993; 100:626-32.

2. Brigden ML, Page NE, Graydon C. Evaluation of the Sysmex NE-8000. Am J Clin Pathol 1993; 100:618-25.

3. Buttarello M, Gadotti M, Lorenz C, Toffalori E, Ceschini N, Valentini $A$, et al. Evaluation of four automated hematology analysers. Am J Clin Pathol 1992; 97:345-52.

\section{Reference range}

Different sources in the literature describe considerably different reference ranges for monocytes. This is most likely due to the different methodologies used for monocyte determination or to different sample populations studied. Comparing the reference range we established for the monoclonal antibody-based flow cytometric method revealed that the upper limits were in fair agreement with those of other reports, while our lower limits were higher $(28,29)$. We do not believe that there really is a different reference range for monocytes for the flow cytometric method because it correlated very well with the microscopic result. It seems more likely that the imprecision of the methods used in other studies (manual techniques or automated haematology analysers) affected their assessment of the reference range. High imprecision of the analytical method especially affects results in the lower monocyte range and inevitably leads to a wider normal range (30).

The difference of monocyte levels between males and females, which was observed in the present study, is consistent with a previous report (29) in which a very large sample population was studied. It is due to the high precision of the flow cytometric method that we could detect this difference despite a relatively small sample number. Our study provides no explanation for the observed sex differences, but the data point to the need for sex-specific reference ranges for the monocyte count.

In summary, flow cytometry based on flurescence-labelled monoclonal antibodies overcomes the main problems of microscopic monocyte counting by its outstanding precision and objective results. The fact that flow cytometry also correlates excellently with the microscopic method apparently makes it an ideal alternative to the 400-cell differential as reference method for monocyte counting. However, as small influences of sample preparation on flow cytometric leukocyte differential results have been reported $(15,20,31)$ a standard sample preparation protocol will be necessary.

4. Devreese K, De Logi E, Francart C, Heyndrickx B, Philippe J, Leroux-Roels G. Evaluation of the automated haematology analyser Sysmex NE-8000. Eur J Clin Chem Clin Biochem 1991; 29:339-45.

5. Drayson RA, Hamilton MSH, England JM. A comparison of differential white cell counting on the Coulter VCS and the Technicon $\mathrm{H} 1$ using simple and multiple regression analysis. Clin Lab Haematol 1992; 14:293-305. 
6. Hallawell R, O'Malley C, Hussein D, Dauer RJ, Tanti M, Wootton AM, et al. An evaluation of the Sysmex NE-8000 hematology analyser. Am J Clin Pathol 1991; 96:594-601.

7. Swaim WR. Laboratory and clinical evaluation of white blood cell differential counts. Am J Clin Pathol 1991; 95:381-8.

8. Warner BA, Reardon DM. A field evaluation of the Coulter STKS. Am J Clin Pathol 1991; 95:207-17.

9. Goosens W, Van Hove L, Verwilghen RL. Monocyte counting discrepancies in results obtained with different automated instruments. J Clin Pathol 1991; 44:224-7.

10. Stølsnes B, Skare EV, Taverner P. A comparison of the differential white blood cell count using the Technicon $\mathrm{Hl}$, the Technicon H6000 and the Coulter VCS. Eur J Haematol 1990; 45 Suppl 53:38-40.

11. Breakall ES, Marchand A, Marcus R, Simson E. Comparison of performance for leukocyte differential counting of the Technicon $\mathrm{H} 6000$ system with a manual reference method using the NCCLS standard. Blood Cells 1985; 11:257-79.

12. National Committee for Clinical Laboratory Standards. Approved Standard H20-A. Reference leukocyte differential count (proportional) and evaluations of instrumental methods. Villanova (PA): National Committee for Clinical Laboratory Standards; 1992.

13. Koepke JA, Dotson MA, Shifman MA. A critical evaluation of the manual/visual differential leukocyte counting method. Blood Cells 1985; 11:173-86.

14. Rümke CL. The statistically expected variability in differential leukocyte counting. In: Koepke JA, editor. Differential leukocyte counting. Skokie (IL): College of American Pathologists, 1978:39-45.

15. Hübl W, Hauptlorenz S, Tlustos L, Jilch R, Fischer M, Bayer PM. Precision and accuracy of monocyte counting. Am J Clin Pathol 1995; 103:167-70.

16. Seaberg R, Cuomo J. Assessment of monocyte counts derived from automated instruments. Lab Med 1993; 24:222-4.

17. Passing $H$, Bablok W. A new biometrical procedure for testing the equality of measurements from two different analytical methods. J Clin Chem Clin Biochem 1983; 21:709-20.

18. Passing $\mathrm{H}$, Bablok W. Comparison of several regression procedures for method comparison studies and determination of sample sizes. J Clin Chem Clin Biochem 1984; 22:431-45.

19. Motulsky HJ, Ransnas LA. Fitting curves to data using nonlinear regression: a practical and nonmathematical review. FASEB J 1987; 1:365-74.

20. Hübl W, Tlustos L, Erath A, Andert S, Bayer PM. Proposed reference method for peripheral blood monocyte counting using fluorescence labelled monoclonal antibodies. Cytometry (Communications in Clinical Cytometry). In press.

21. Linnet $\mathrm{K}$. Evaluation of regression procedures for method comparison studies. Clin Chem 1993; 39:424-32.

22. Combleet PJ, Myrick D, Levy R. Evaluation of the Coulter STKS five-part differential. Am J Clin Pathol 1993; 99:72-81.

23. Johannessen $B$, Ommundsen $T$, Minde TE, Haneberg $B$, Sandberg S. Automated differential leukocyte counts in newborn infants. Comparison of Coulter VCS and Technicon $\mathrm{H} 1$ with manual counts. Eur J Haematol 1990; 45 Suppl 53:41-4.

24. Bentley SA, Johnson TS, Sohier CH, Bishop CA. Flow-cytochemical differential leukocyte analysis with quantitation of neutrophil left shift. Am J Clin Pathol 1994; 102:223-30.

25. Bas BM, Catsberg MJ, Op de Kamp SLK. A short evaluation of a new haematological analyser: the Cobas Argos 5 Diff. Eur J Clin Chem Clin Biochem 1993; 31:603-8.

26. Avenarius HJ, Deinhardt J. The monocytes: their recognition by different techniques. Laboratoriumsmedizin 1992; 16:204-9.

27. Lebeck LK, Mast BJ, Houwen B. Flow cytometric white blood cell differentials. A proposed alternate reference method. Sysmex J Int 1993; 3:61-9.

28. Kjeldsberg $\mathrm{C}$. Normal blood and bone marrow values in man. In: Lee GR, Bithell TC, Foerster J, Athens JW, Lukens JN, editors. Wintrobe's clinical hematology. 9th ed. Malvern (PA): Lea \& Febiger, 1993:2297-309.

29. Saxena S, Wong ET. Heterogeneity of common hematologic parameters among racial, ethnic and gender subgroups. Arch Pathol Lab Med 1990; 114:715-9.

30. Henry RJ, Reed AH. Normal values and the use of laboratory results for the detection of disease. In: Henry RJ, Cannon DC, Winkelmann $\mathrm{JW}$, editors. Clinical chemistry, principals and technics. 2nd ed. Hagerstown (ML): Harper \& Row, 1974:343-71

31. Serke S, Huhn D. An all leukocyte whole blood lyse method for multiparameter flow-cytometry. Clin Lab Haematol 1993; 15:275-85.

Dr. Wolfgang Hübl

Institut für Klinische Chemie

Wilhelminenspital

Montlearstraße 37

A-1171 Wien

Austria 


\section{।}

: 